\title{
Forced Convection through Discrete Heat Sources and Simple Thermal Model - A Numerical Study
}

\author{
Kartikaswami Hasavimath \\ Department of Studies in Mechanical Engineering, \\ University B. D. T. College of Engineering, Davanagere -577004, Karnataka, India \\ Kishan Naik \\ Department of Studies in Mechanical Engineering, \\ University B. D. T. College of Engineering, Davanagere -577004, Karnataka, India \\ Banjara Kotresha \\ Department of Mechanical Engineering, \\ National Institute of Technology Karnataka, Surathkal - 575025, Karnataka, India \\ N. Gnanasekaran \\ Department of Mechanical Engineering, \\ National Institute of Technology Karnataka, Surathkal - 575025, Karnataka, India \\ Corresponding author: bkotresha@gmail.com ; gnanasekaran@nitk.edu.in
}

(Received January 5, 2019; Accepted August 22, 2019)

\begin{abstract}
In this work a forced convection through discrete heat sources and simple thermal model placed inside the vertical channel is analyzed numerically. The problem considered for the investigation comprises of a vertical channel with distinct heat source assembly located at the center of the channel. The novelty of the present work is to replace the discrete heat source assembly by a simple thermal model to obtain uniformly distributed temperature and streamlines. A conjugate heat transfer investigation is carried out because the problem domain consists of aluminum solid strips as well as Bakelite strips in discrete heat source assembly which are replaced by a aluminum solid in case of simple thermal model. The numerically obtained data are initially compared with experimental data for the purpose of validation. The temperature of each discrete sources decrease with increase in inlet velocity of the fluid and bottom heat source is able to take higher heat load. The results in terms excess temperature obtained for both discrete heat source and simple thermal model is presented and discussed.
\end{abstract}

Keywords- Vertical channel, Mixed convection, Simple thermal model, Discrete heat sources.

\section{Introduction}

The rapid development of electronic industries and reduced dimension of electronic components results in less space for excessive heat dissipation. Hence, the cooling of electronic elements became essential for the improved life of the components, noteworthy operation and for its safety. The natural convection is most preferred as the cooling method in the applications where the heat flux is lower than $10 \mathrm{~W} / \mathrm{cm}^{2}$. But for higher heat flux generating equipment's the forced convection is preferred as the cooling method using air or water as the fluid. Mixed convection is most suitable to overcome the forced convection problem in some extent and plays an important role in the advantages of both natural and forced convection. Many researchers extensively investigated mixed convection through channels, pipes/tubes and ducts because of its appearance in the variety of engineering applications. 
International Journal of Mathematical, Engineering and Management Sciences

Vol. 4, No. 6, 1397-1406, 2019

https://dx.doi.org/10.33889/IJMEMS.2019.4.6-110

There are number of studies performed for heat transfer in horizontal, vertical and inclined channels by many researchers. Chen and Chung (1996) analytically performed the linear stability of mixed convection heater transfer in a vertical channel. The side wall of the channel is defined with heat and which in turn varies the temperature in the vertical channel. They analysed that the maximum amplifications rates for both gravity assisted and opposed flows was found insensitive for different temperature perturbations. Hadim and Chen (1994) performed a mixed convection investigation through discrete heat sources with porous medium attached on the left wall in a vertical channel. The study reveals that the separation point is same for different Darcy numbers but the reattachment point moves downwards. They also conclude that the increase in Darcy number increases the average Nusselt number.

Premachandran and Balaji (2006) numerically investigated heat transfer through extended heat sources in a channel by keeping the size of channel, thickness of heat sources and spacing between the heaters as constant. They found that maximum temperature and effect of radiation decreases as Reynolds number increases. Hotta et al. (2014) conducted steady state experiments with five discrete sources under mixed convection cooling and found that as $\lambda$ value (heuristic non-dimensional geometric distance parameter) increases the maximum temperature decreases and the optimal configuration occurs for the highest value of $\lambda$. Gururaja Rao et al. (2002) numerically calculated mixed convection through a vertical channel in which the flush mounted heat sources are kept in the walls. The study includes the surface radiation emitted by the heat sources and concludes that the surface emissivity plays important role in heat transfer. Chen et al. (2013) performed analysis of forced convection in metal foams placed in a horizontal channel with distinct heaters placed on the bottom wall of the channel. They found that the increasing the heat exchange between solid and fluid phases at the interface results in reduction in the excess temperature between the solid and fluid phases at a particular velocity. Sankar et al. (2013) computationally examined the natural convection through porous medium filled in a vertical channel saturated by fluid with discrete heat sources. The result discloses that the heat transfer rate enhances with increase in Darcy and Rayleigh numbers. It is also presented that the length of the heaters also influences on the maximum temperature and heat transfer in the annular cavity.

Hotta et al. (2013) conducted experiments on protruding distinct heat sources to see the effect of surface radiation by natural convection. They reported that the uppermost heat generating source should be placed at the bottom and they achieved $12 \%$ increase in heat transfer by applying black paint on the sources. Ghorab (2015) investigated numerically forced convection through heat exchanger for non-porous and porous medium partly filled in channels with changing the exit height. The result reports that temperature decreases with decrease in exit height. They also concluded that the overall thermal performance decreases with increasing Reynolds number because of increase in pumping power. Kamath et al. (2014) conducted experiments on highly porous metallic foams placed in a vertical channel. They studied different pore density metal foams with different porosities. The result reveals that the higher pores per inch metal foam gives highest heat transfer at the expense of pressure drop. Kotresha and Gnanasekaran (2019) numerically calculated the forced convection through discrete heat sources attached with metallic foams in a vertical channel. They considered two dissimilar types of metal foam material for the investigation. They concluded that excess temperature decreases for all the heat sources with increase in air inlet velocity and bottom heat source can take higher heat load. They found that the copper metal foam shows a less improvement in heat transfer which is not significant compared to aluminium metallic foam. The isothermal condition on all the heaters is achieved at a particular velocity for both aluminium and copper metal foams. Ahamad and Balaji (2015) studied laminar conjugate mixed convection through distinct heater sources by applying simple thermal model 
International Journal of Mathematical, Engineering and Management Sciences

Vol. 4, No. 6, 1397-1406, 2019

https://dx.doi.org/10.33889/IJMEMS.2019.4.6-110

concept. They revealed that the geometric complexity can be reduced by applying simple thermal model concept instead of discrete heat sources which result in uniformly distributed heat in the domain.

Based on the above literature, it is found that the many experimental and numerical studies are carried out on channels by considering different types of heat transfer enhancement techniques. Even though there are numerous studies on discrete heat sources placed inside the channels, the authors found very limited study on simple thermal model in the literature. Hence, this work concentrates on fluid flow and temperature distribution in the channel for both discrete heat sources and simple thermal model. The results in terms of temperature contour, excess temperature obtained are presented. The application of the present computational geometry considered resembles the heat sources present in a printed circuit board of electronic components.

\section{Physical Geometry}

The geometry considered consists of a distinct heater assembly positioned at the centre of the vertical channel as shown in Figure 1. The discrete heater plate assembly is a combination of aluminium strips and Bakelite strips. The aluminium as well as Bakelite strips are placed one after the other to make the assembly. A heater is placed in between two aluminium strips and acts as heat source and three aluminium heat sources are kept in the discrete heater assembly. The size of the aluminium and Bakelite strips is $250 \times 20 \times 3$ (all in mm) and $250 \times 22.5 \times 7$ (all in mm).

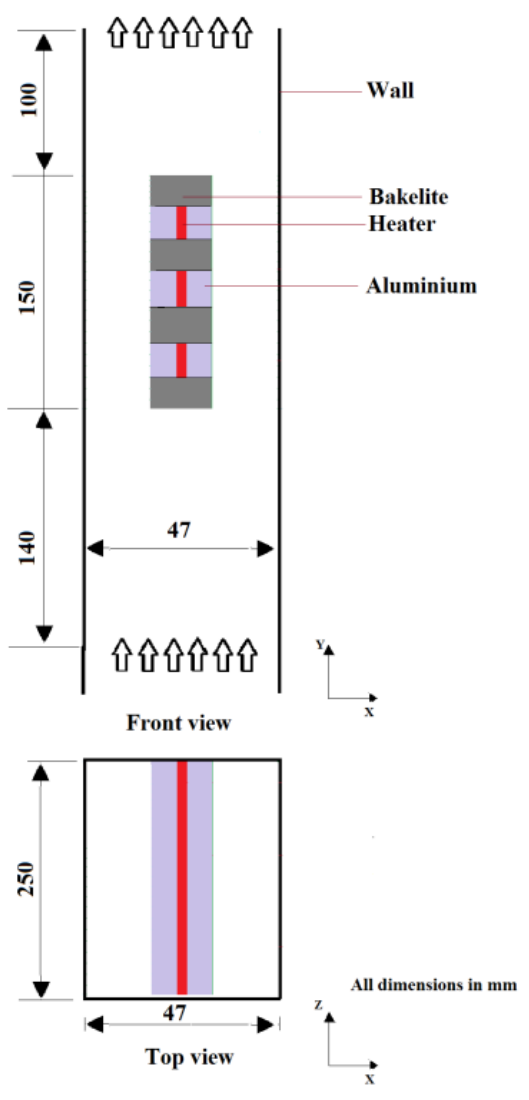

Figure 1. Physical domain of the vertical channel 
International Journal of Mathematical, Engineering and Management Sciences

Vol. 4, No. 6, 1397-1406, 2019

https://dx.doi.org/10.33889/IJMEMS.2019.4.6-110

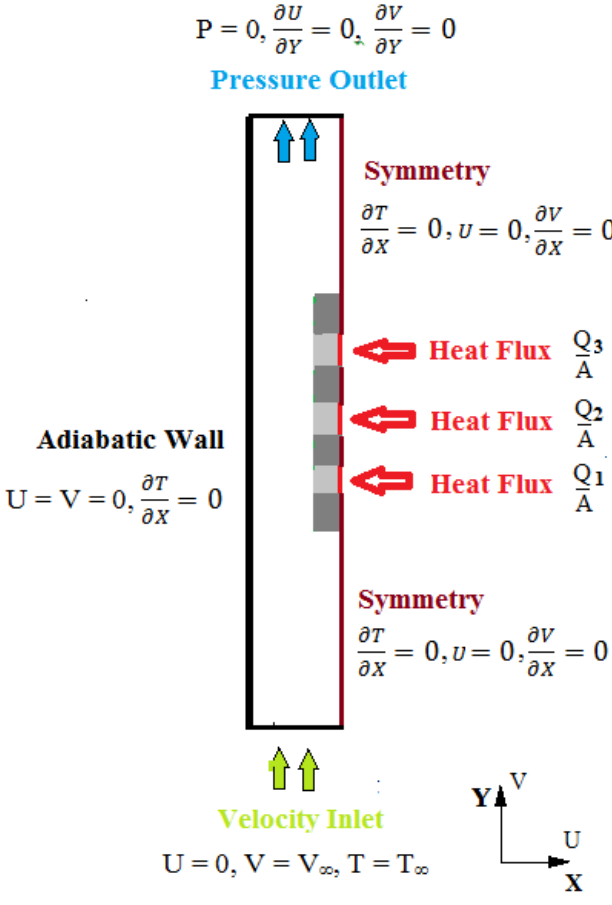

Discrete heat sources

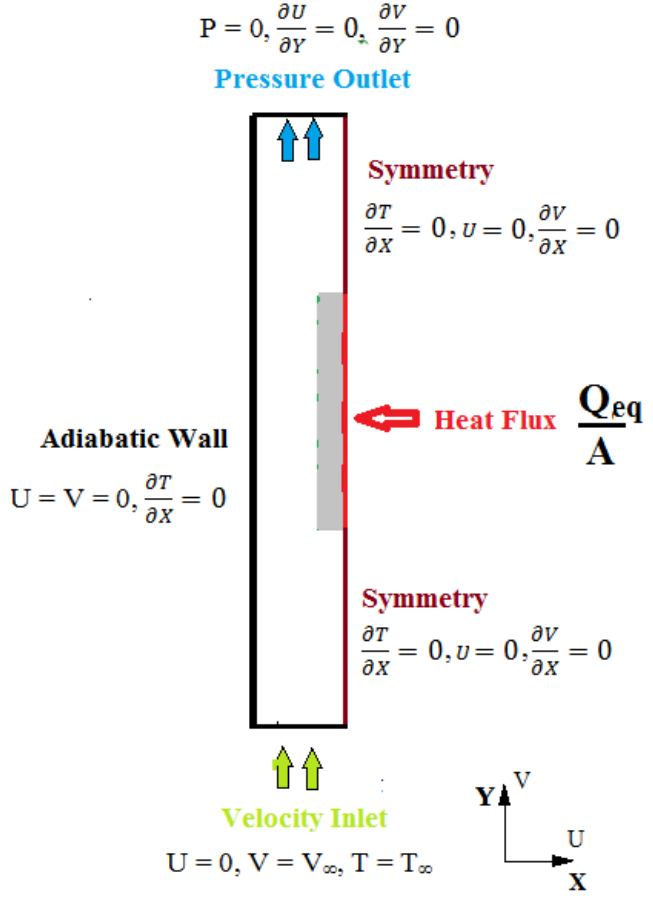

Simple thermal model

Figure 2. Boundary conditions on the computational domain

\section{Boundary Conditions}

The numerical computation is performed for one half of the geometry due to its symmetry about vertical axis. Subsequently, one side of the channel with half of the aluminium discrete heater plate are considered for further analysis. In boundary conditions, a uniform velocity and zero pressure is assigned at inlet and outlet of the channel respectively. A known heat flux is specified for the heater and the side wall is kept adiabatic. The boundary conditions on the computational domain are shown in Figure 2.

\section{Simulation Details}

The numerical simulations are performed on the selected computational domain using commercially available ANSYS FLUENT. The governing equations used in the open area of the channel are similar to pipe flow. The air flows through the vertical channel with temperature of $30^{\circ} \mathrm{C}$. The air velocity at the inlet is varied from 0.42 to $3.5 \mathrm{~m} / \mathrm{s}$ and the hydraulic diameter based Reynolds number varies from 2000 to 17000 . The turbulent flow characteristic is captured using $\mathrm{k}-\omega$ turbulence model. A conjugate heat transfer study is carried out for the computational domain since it involves both fluid as well as solid domains. The governing equations of continuity, momentum and energy considered for the channel are given in Eq. $(1-5)$.

Continuity equation:

$\frac{\partial u}{\partial x}+\frac{\partial v}{\partial y}=0$ 
International Journal of Mathematical, Engineering and Management Sciences

Vol. 4, No. 6, 1397-1406, 2019

https://dx.doi.org/10.33889/IJMEMS.2019.4.6-110

Momentum equations:

$u \frac{\partial u}{\partial x}+v \frac{\partial u}{\partial y}=-\frac{1}{\rho} \frac{\partial p}{\partial x}+\mu\left(\frac{\partial^{2} u}{\partial x^{2}}+\frac{\partial^{2} u}{\partial y^{2}}\right)$

$u \frac{\partial v}{\partial x}+v \frac{\partial v}{\partial y}=-\frac{1}{\rho} \frac{\partial p}{\partial y}+\mu\left(\frac{\partial^{2} v}{\partial x^{2}}+\frac{\partial^{2} v}{\partial y^{2}}\right)+g \beta\left(T-T_{\infty}\right)$

Energy Equation for fluid:

$u \frac{\partial T}{\partial x}+v \frac{\partial T}{\partial y}=\alpha\left(\frac{\partial^{2} T}{\partial x^{2}}+\frac{\partial^{2} T}{\partial y^{2}}\right)$

For solid aluminium plates

$\nabla \cdot\left(k_{s} \nabla T\right)=0$

The pressure and velocity are coupled using coupled scheme with pseudo transient in time. A second order upwind scheme is used for pressure, velocity, energy and for turbulence parameters. The convergence criteria for continuity are set below $1 \times 10^{-5}$, momentum is $1 \times 10^{-5}$, energy is $1 \times 10^{-10}$ and turbulence parameters it is $1 \times 10^{-3}$.

\section{Simple Thermal Model}

The concept of simple thermal model proposed by Ahamad and Balaji (2015) is used in the present study by replacing the discrete heaters by a uniform heat generating volume. The thickness of simple thermal model is same as that of discrete heat sources. The net heat input to the heater for simple thermal model is given in Eq. (6)

$q_{e q}=\frac{\sum_{i=1}^{n} q_{i} A_{i}}{A_{e q}}$

\section{Results and Discussion}

\subsection{Grid Independence Study:}

In the present computation the mesh for the selected numerical domain is created using ANSYS workbench 15.0. Quad cells of three different grid sizes are considered for the grid sensitivity analysis. A boundary layer mesh is used near the wall and near the discrete heat sources as well as near the simple thermal model. Table 1 shows the grid independency results carried out in the present study, based on the results the mesh size of 78,614 is selected as optimum for further numerical predictions because it gives less pressure deviation.

Table 1. Grid independence study

\begin{tabular}{ccccc}
\hline Grid Size & $\begin{array}{c}\text { Maximum pressure } \\
(\mathrm{Pa})\end{array}$ & $\begin{array}{c}\text { Maximum temperature } \\
(\mathrm{K})\end{array}$ & Pressure & $\begin{array}{c}\text { Deviation } \\
\text { Temperature }\end{array}$ \\
\hline 41,506 & 0.129 & 370 & 11.0344 & 0 \\
78,614 & 0.14 & 370 & 3.448 & 0 \\
$1,12,668$ & 0.145 & 370 & \multicolumn{2}{c}{ Baseline } \\
\hline
\end{tabular}


International Journal of Mathematical, Engineering and Management Sciences

Vol. 4, No. 6, 1397-1406, 2019

https://dx.doi.org/10.33889/IJMEMS.2019.4.6-110

\subsection{Validation of Numerical Results}

The present computational data are compared with experimental data available in the literature for validating of the methodology adopted in the study. The excess temperature is computed as the difference in temperature of the heater and ambient temperature. For the purpose of validation the excess temperature calculated for the bottom heater is compared with the data of Kamath et al. (2014) and is shown in Figure 3. It is clear that the numerical result matches fairly well with the experimental results. This comparison proves that the solution methodology adopted in the present analysis is correct.

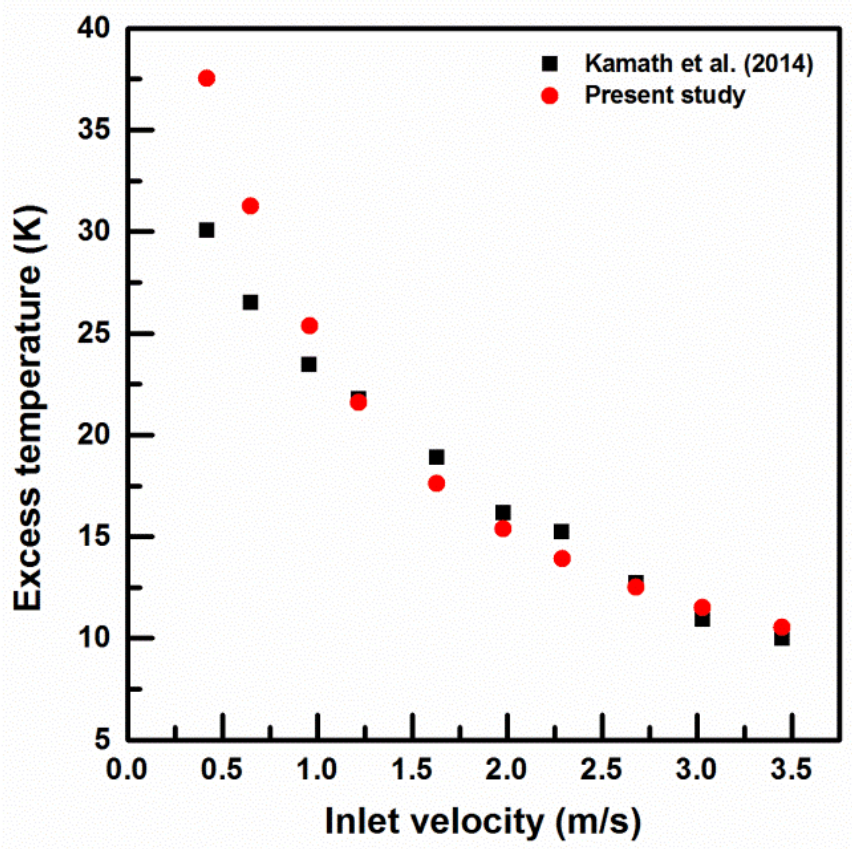

Figure 3. Variation of bottom heater temperature

\subsection{Thermal Results}

Figure 4 and Figure 5 shows the variation of temperature in the channel for both discrete heat source and simple thermal model. The top heater receives higher heat in the case of discrete heat source since the air moving towards top carries heat from the bottom as well as middle heaters. The temperature distribution in the channel is not uniform but case of whereas in simple thermal model the temperature distribution is uniform through the aluminium strip. The excess temperature in the channel is less in the case of simple thermal model compared to discrete heat source. This confirms that the discrete heat source chips in the electronic components can be replaced by uniform generating chips for the smooth working of the components. 
International Journal of Mathematical, Engineering and Management Sciences

Vol. 4, No. 6, 1397-1406, 2019

https://dx.doi.org/10.33889/IJMEMS.2019.4.6-110

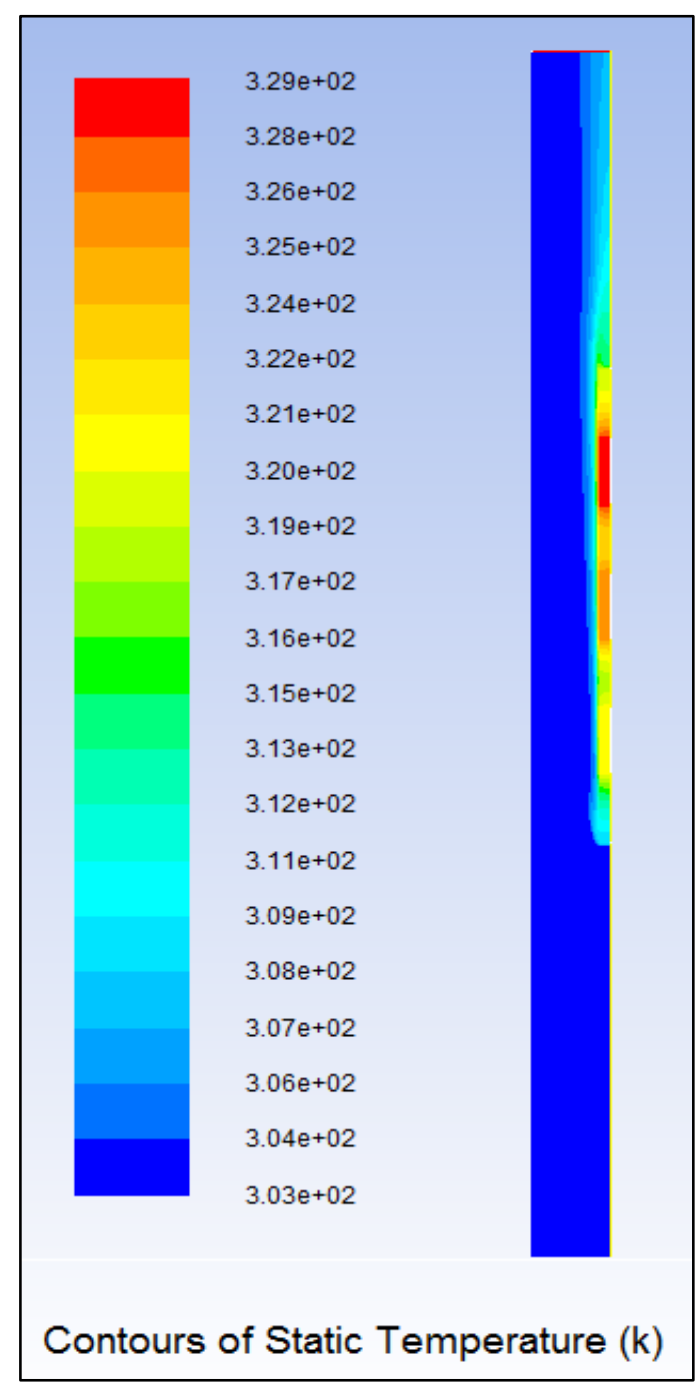

Figure 4. Temperature contours for discrete heat sources on vertical channel

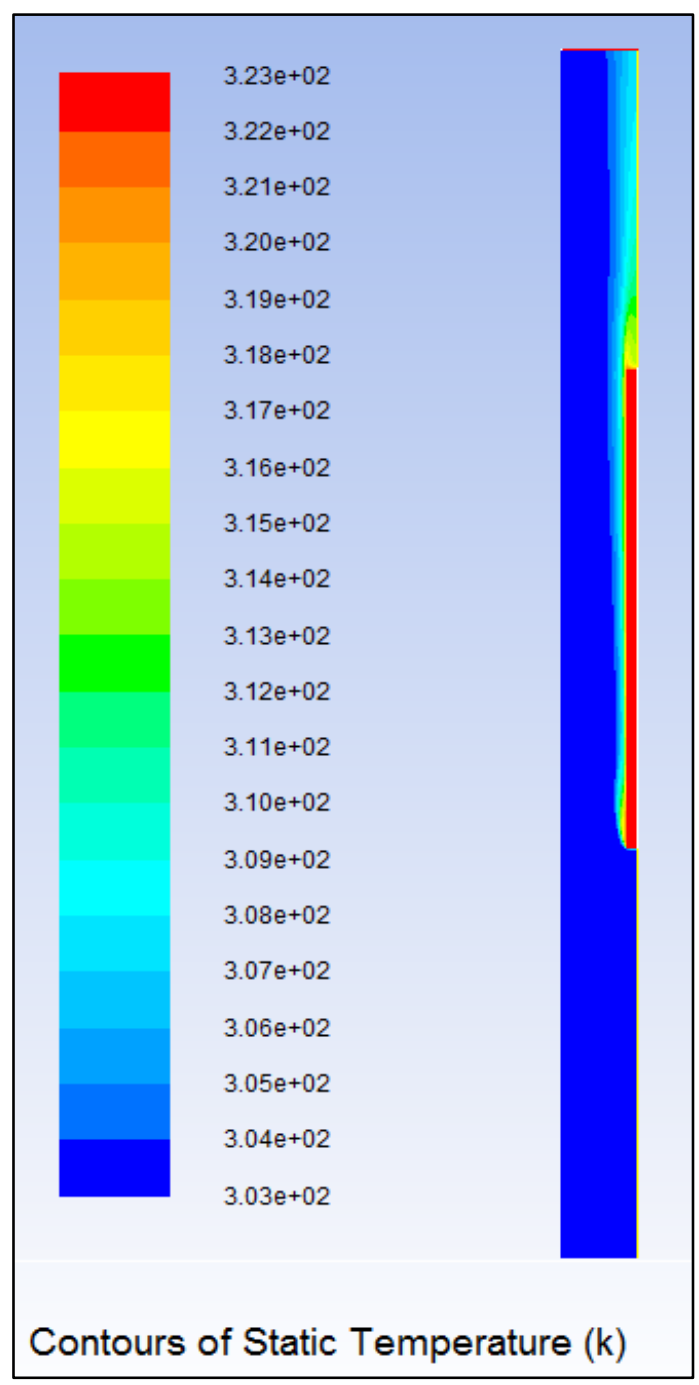

Figure 5. Temperature contours for simpler thermal model

The excess temperature variation with Reynolds number for discrete heaters and simple thermal model is shown in Figure 6 and Figure 7 respectively. The excess temperature decreases with increase in the Reynolds number for both scenarios. The bottom heater in the discrete heater assembly can take higher heat load since it shows less excess temperature. The excess temperature obtained in the simple thermal model almost lies between of excess temperature obtained at the middle heater and bottom heater. 
International Journal of Mathematical, Engineering and Management Sciences

Vol. 4, No. 6, 1397-1406, 2019

https://dx.doi.org/10.33889/IJMEMS.2019.4.6-110

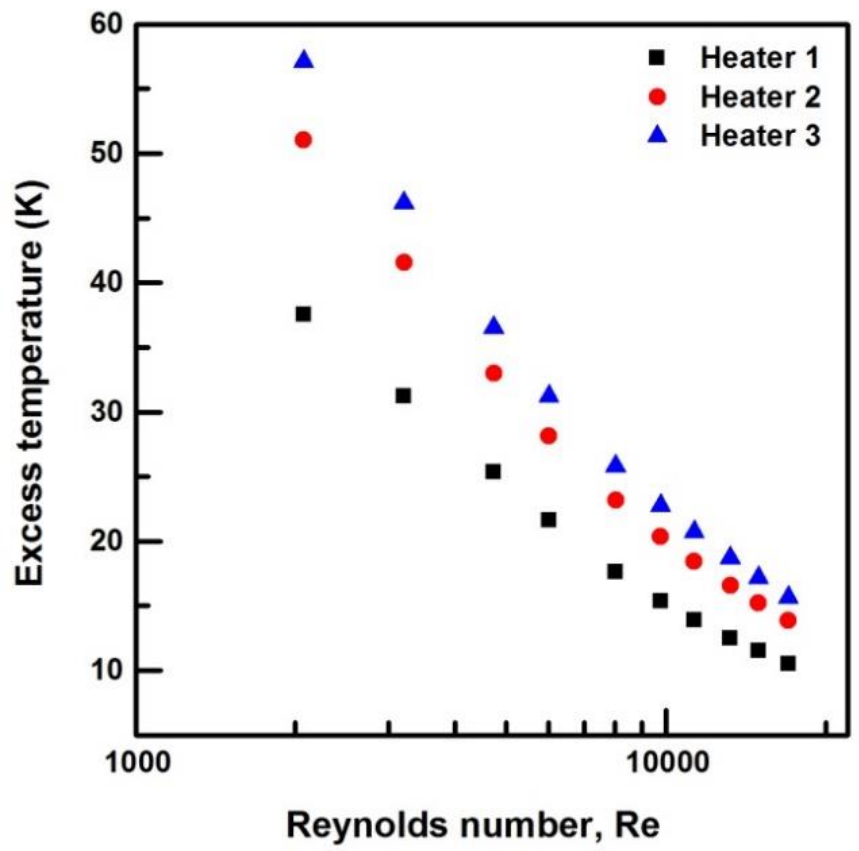

Figure 6. Variation of excess temperature for discrete heat sources

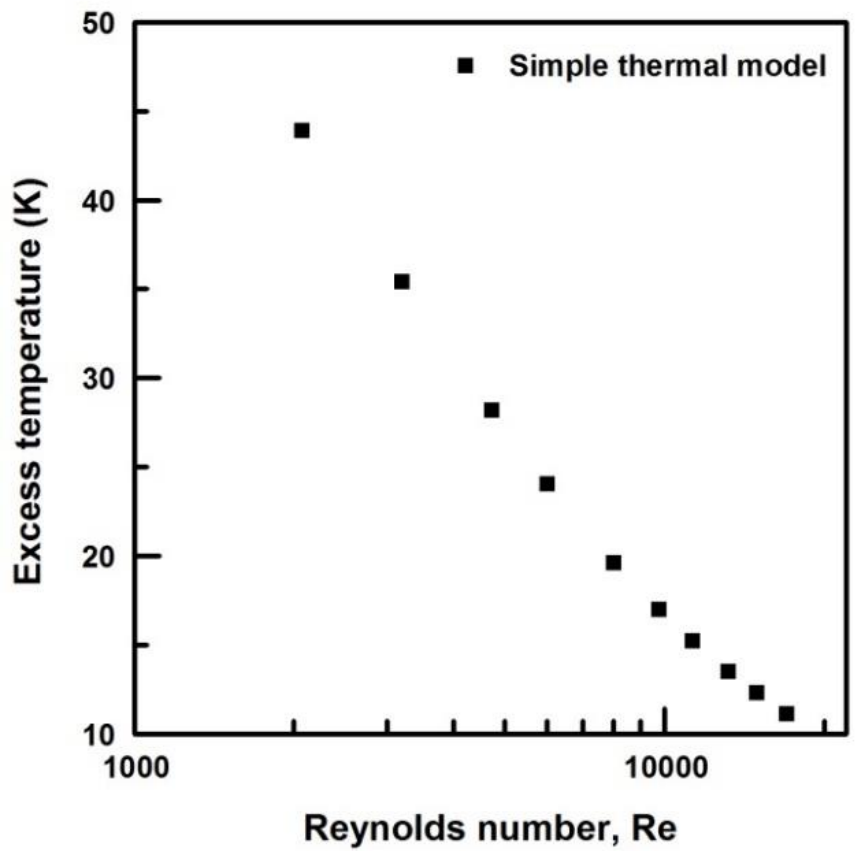

Figure 7. Excess temperature variation for simple thermal model 
International Journal of Mathematical, Engineering and Management Sciences

Vol. 4, No. 6, 1397-1406, 2019

https://dx.doi.org/10.33889/IJMEMS.2019.4.6-110

\section{Conclusions}

A two-dimensional computational examination is performed on discrete heaters and simple thermal model placed at the centre of the vertical channel. The discrete heater assembly is combination of aluminium - Bakelite strips and only aluminium strip is used in the simple thermal model. The numerical simulations are performed for a range of Reynolds number by assigning equal heat input to the heaters. The experimental and numerical data are compared initially for validating of numerical methodology adopted in the present study. The silent conclusions based on the study are

- The excess temperature obtained in the simple thermal model is less compared to discrete heat sources.

- The simple thermal model shows a uniform heat distribution in the channel but whereas temperature distribution is not uniform in the case of discrete heat sources.

- The discrete heat sources can be replaced by a simple thermal model which gives less temperature and uniform temperature profiles.

\section{Conflict of Interest}

The authors confirm that there is no conflict of interest to declare for this publication.

\section{Acknowledgement}

The authors would like to express their sincere thanks to the referees and for their valuable suggestions towards the improvement of the paper and also the CMFDP-2019 conference coordinators.

\section{References}

Ahamad, S.I., \& Balaji, C. (2015). A simple thermal model for mixed convection from protruding heat sources. Heat Transfer Engineering, 36(4), 396-407.

Chen, C.C., Huang, P.C., \& Hwang, H.Y. (2013). Enhanced forced convective cooling of heat sources by metal-foam porous layers. International Journal of Heat and Mass Transfer, 58(1-2), 356-373.

Chen, Y.C., \& Chung, J.N. (1996). The linear stability of mixed convection in a vertical channel flow. Journal of Fluid Mechanics, 325, 29-51.

Ghorab, M.G. (2015). Forced convection analysis of discrete heated porous convergent channel, Heat Transfer Engineering, 36(9), 829-846.

Gururaja Rao, C., Balaji, C., \& Venkateshan, S.P. (2002). Effect of surface radiation on conjugate mixed convection in a vertical channel with a discrete heat source in each wall. International Journal of Heat and Mass Transfer, 45(16), 3331-3347.

Hadim, A., \& Chen, G. (1994). Non-darcy mixed convection in a vertical porous channel with discrete heat sources at the walls. International Communications in Heat and Mass Transfer, 21(3), 377-387.

Hotta, T.K., Balaji, C., \& Venkateshan, S.P. (2014). Optimal distribution of discrete heat sources under mixed convection - a heuristic approach. Journal of Heat Transfer, 136(10), 104503-5.

Hotta, T.K., Muvvala, P., \& Venkateshan, S.P. (2013). Effect of surface radiation heat transfer on the optimal distribution of discrete heat sources under natural convection. Heat and Mass Transfer, 49(2), 207-217. 
International Journal of Mathematical, Engineering and Management Sciences

Vol. 4, No. 6, 1397-1406, 2019

https://dx.doi.org/10.33889/IJMEMS.2019.4.6-110

Kamath, P.M., Balaji, C., \& Venkateshan, S.P. (2014). Heat transfer enhancement with discrete heat sources in a metal foam filled vertical channel. International Communications in Heat and Mass Transfer, 53, 180-184.

Kotresha, B., \& Gnanasekaran, N. (2019). A synergistic combination of thermal models for optimal temperature distribution of discrete sources through metal foams in a vertical channel. Journal of Heat Transfer, 141(2), 022004 (1-8).

Premachandran, B., \& Balaji, C. (2006). Conjugate mixed convection with surface radiation from a horizontal channel with protruding heat sources. International Journal of Heat and Mass Transfer, 49(19-20), 3568-3582.

Sankar, M., Park, J., Kim, D., \& Do, Y. (2013). Numerical study of natural convection in a vertical porous annulus with an internal heat source: effect of discrete heating. Numerical Heat Transfer-Part A, 63(9), $687-712$. 